\title{
ESTRATEGIAS FENOMORFOLÓGICAS DE ESPECIES DE UN MATORRAL MEDITERRÁNEO (ANDALUCÍA, ESPAÑA)
}

\author{
Teresa NAVARRO y Baltasar CABEZUDO
}

\begin{abstract}
RESUMEN. Estrategias fenomorfológicas en especies de un matorral mediterráneo (Andalucía, España). Se ha estudiado la fenomorfología de 32 plantas perennes de una comunidad vegetal, situada en el S de España (Sicrra de Mijas, Málaga) en la que podemos reconocer distintas etapas dinámicas: coscojar, aulagar-jaral y tomillar. Se ha comparado la dinámica del crecimiento vegetativo entre las diferentes especies. Las especies de la comunidad muestran una fenología asincrónica respecto a la actividad vegetativa (crecen durante todo el año, con un máximo en primavera y otro en otoño) y a la floración (con un máximo en primavera y otros dos, inferiores en verano y otoño), relacionada con las estrategias adaptativas de modulación del crecimiento de las especies respecto al clima mediterráneo (sequía estival). Se reconocen cuatro estrategias adaptativas: a. Siempreverdes esclerófilas, con rápido crecimiento vegetativo, elongación y floración en abril-mayo, previo al inicio del período de sequía. b. Decíduas de otoño, con actividad vegetativa y reproductiva centrada en la primavera. c. Decíduas de verano, con actividad vegetativa en otoño, invierno y primavera, floración en primavera e inactivas, arrojando todas las hojas y ramas verdes, durante la sequía estival. d. Semidecíduas de verano, crecen durante todo el año, con dos períodos de máxima actividad vegetativa (primavera y otoño) separados por un ralentizamiento del crecimiento en el período de sequía; floración básicamente primaveral, pudiendo extenderse al verano y otoño.
\end{abstract}

Palabras clave. Fenomorfología, clima mediterráneo, matorrales mediterráneos (maquia y garriga), estrategias adaptativas.

ABSTRACT. Phenomorphological strategies of Mediterranean shrub-land species (Andalusia, Spain). A phenomorphological study was carried out in southern Spain (Sierra de Mijas, Málaga) in order to recognise the dynamic stages of a community compounded by 32 shrub Mediterranean species. We have recognised two different dynamic stages: maquis and garriga and the growth activity among the different species have been compared. The plant community shows an asynchronous phenological trend as regard as the vegetative activity (growing throughout the year with a peak in Autumn and Spring) and flowering (with a maximum peak during Spring and two lower ones in Autumn and early Summer). This is in relation with their adaptation strategies in growth activity modulation due to the Mediterranean climate (Summer drought). The results show four different groups: a. Evergreen sclerophyllous species limiting their growth activity and flowering to a brief period before the dry stress (April-May). b. Autumn-deciduous with vegetative and reproductive activities limited to Spring. c. Summer-deciduous with vegetative activity in Autumn and Winter, flowering in Spring and stops in the dry season shedding their leaves and shoots. d. Summersemideciduous species, growing throughout the year, with maximum vegetative activity in Spring and Autumn and diminishing in the cold season. The peak of flowering takes place in Spring, but extends to Summer and Autumn.

Key words. Phenomorphology, Mediterranean shrubland species, adaptative strategies.

Trabajo realizado gracias a la ayuda concedida por la Junta de Andalucía al grupo de investigación RMN-l15 de la Universidad de Málaga. 


\section{INTRODUCCIÓN}

Se considera actualmente que los estudios fenológicos de comunidades vegetales en el Mediterráneo son escasos (Bergmeier \& Matthäs, 1996). Destacan los realizados en Grecia (Arianoutsou-Faraggitaki \& Diamantopoulos, 1985; Diamantopoulos \& Margaris, 1981 y Bergmeier, 1998), Israel (Orshan, 1989) e Italia (de Lillis \& Fontanella, 1992 y Gratani \& Crescente, 1997). En la Península Ibérica, se han realizado estudios sobre comunidades portuguesas (Correira et al., 1992) y en el sur de España sobre el comportamiento fenológico de comunidades de alcornocales (Cabezudo et al., 1993 y Peréz Latorre et al., 1995), badlands (Navarro et al., 1993), vegetación litoral (Herrera, 1986; Arroyo, 1988; Laguna et al., 1986 y Moreno et al., 1997) y floración de matorrales termófilos (Cabezudo et al., 1992 e Hidalgo \& Cabezudo, 1994). Sin embargo, no ha sido estudiado el comportamiento fenológico completo de un típico matorral basófilo y termófilo, integrante del monte mediterráneo, que permita un estudio comparativo de los patrones fenológicos y estrategias de comportamiento con otros matorrales (maquias y garrigas) del Mediterráneo occidental.

El clima mediterráneo se caracteriza porque el verano es la estación cálida y seca, y en consecuencia el período donde las plantas acusan una efectiva sequía fisiológica (Quezel, 1985). En el clima mediterráneo, el ciclo de crecimiento estacional para la mayor parte de los arbustos siempreverdes se realiza al inicio de la primavera (Herrera, 1986; Pérez Latorre et al., 1995; Cabezudo et al., 1993 y IshShalom-Gordon, 1993), siendo en esta estación donde se produce mayoritariamente el crecimiento y la floración (Oechel et al., 1981 y de Lillis \& Fontanella, 1992); por el contrario, en ecosistemas áridos, ambas fases fenológicas se producen fundamentalmente en invierno (Navarro et al., 1993 y Bertiller et al., 1991).
La floración para la mayoría de plantas perennes en zonas de clima mediterráneo, puede extenderse hasta el verano y otoño en condiciones especiales o como estrategias de determinadas especies (Kummerov, 1983). Este tipo de floración multiestacional ha sido observado en comunidades de plantas del sureste árido con prolongados períodos de sequía (Navarro et al., 1993) y de comunidades de dunas costeras (Moreno et al., 1997).

El clima determina las condiciones generales de humedad del suelo y de temperatura, así como la diversidad de formas de crecimiento, como resultado de una estrategia particular para la utilización óptima de los recursos (Cody, 1986). Se ha demostrado que, el comportamiento fenológico de las especies de una comunidad puede ser afectado por factores particulares como tipo de suelo o microtopografía (Bergmeier, 1998), variación de la humedad del suelo (Gómez Sal et al., 1986) efecto del fuego (Trabaud \& de Chanterac, 1985). Como ocurre en ecosistemas áridos (Bertiller et al., 1991), la comunidad puede mostrar una asincronía entre las estrategias de las especies de acuerdo con la potencialidad de cada una para explotar el agua y los nutrientes almacenados en el suelo; esta asincronía puede estar marcada por la adaptación de cada tipo biológico a estrategias funcionales distintas. La co-existencia de plantas con diferentes estrategias adaptativas en un mismo ecosistema depende de la utilización del agua como recurso, en el espacio y tiempo, de la temperatura y de la composición del suelo. La estrategia de modulación del crecimiento adoptada en el período de sequía por las especies mediterráneas es, en caso extremo, evitar la sequedad arrojando las hojas en verano y cesando su crecimiento, o ser tolerantes a la sequía y no cesar su crecimiento durante la estación seca sino ralentizarlo (Montenegro, 1987 y Orshan et al., 1988).

En este trabajo se ha comparado la dinámica de las actividades vegetativas y 
reproductivas de las especies más características de un matorral (maquis) mediterráneo, así como las diferentes respuestas fenomorfológicas en la modulación estacional de su actividad vegetativa, mostrando como, las diferentes etapas dinámicas de la vegetación y las variaciones topográfico-edáficas, segregan diferentes formas de crecimiento y estrategias adaptativas frente al período de sequía.

\section{MATERIAL Y MÉTODOS}

\section{Área de estudio.}

El área de estudio, Sierra de Mijas (UF 5556), se localiza en la provincia de Málaga al SW de la capital, a una altitud media de $500 \mathrm{~m}$ y con sustrato fundamentalmente calizodolomítico. Climáticamente (fig. 1) se encuadra en la región mediterránea, con una corta época de lluvias en otoño-invierno y máxima insolación en verano. El período de aridez se extiende desde finales de mayo a primeros de septiembre. La temperatura media anual es de $17,3{ }^{\circ} \mathrm{C}$ y la precipitación media de $575,4 \mathrm{ml}$. La precipitación mínima anual es en julio $(1,5$ $\mathrm{ml})$, y la máxima en noviembre (106,9 ml). El

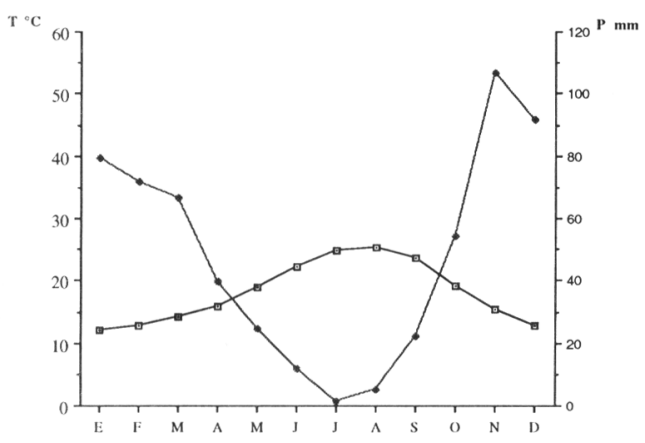

Figura 1. Diagrama ombrotérmico (30 años) de la Sierra de Mijas (Málaga). Hydrothermic diagram (30 years) of Sierra de Mijas (Málaga). mes más cálido es agosto $\left(25,3{ }^{\circ} \mathrm{C}\right)$ y el más frío enero $\left(12^{\circ} \mathrm{C}\right)$.

La vegetación potencial del territorio está representada por un coscojar-encinar (maquia) en forma de bosquetes de fanerófitos y nanofanerófitos. Por degradación del coscojar aparecen comunidades seriales como jaralesaulagares (garriga), formación predominante en la actualidad y tomillares en las zonas con escaso desarrollo edáfico. Las especies con mas cobertura en el área de estudio son Rosmarinus officinalis, Ulex baeticus, Cistus albidus, Cistus clusii, Thymbra capitata, Juniperus oxycedrus subsp. oxycedrus, Quercus ilex subsp. ballota, Stipa tecissima, Quercus coccifera, Cistus monspeliensis, Phlomis purpurea y Lavandula stoechas ( Hidalgo \& Cabezudo, 1994 y Nieto Caldera et al. 1987).

\section{Observaciones fenológicas.}

El seguimiento de las fases fenomorfológicas de las 32 especies perennes estudiadas, se realizó durante el período comprendido entre julio de 1996 y febrero de 1997; las observaciones se realizaron mensualmente, sobre tres individuos de cada especie y sobre seis ramas diferentes de cada individuo. Se siguió la metodología propuesta por Orshan (1989) y desarrollada por nosotros en trabajos anteriores (Cabezudo et al. 1993; Navarro et al., 1993 y Pérez Latorre et al., 1995). Para la observación de la actividad vegetativa seguimos las fases propuestas por Bertiller et al. (1991) que incluye el crecimiento vegetativo de los dolicoblastos y braquiblastos, desde que son visibles las primeras hojas que emergen hasta que cesa su actividad, incluyendo el período de elongación de los entrenudos en los tallos y ramas. Para el diagrama de funcionamiento de la comunidad se tuvieron en cuenta los porcentajes de especies que en cada período mensual presentaban la misma fase fenológica. Los tipos 
biológicos fueron designados en base al sistema de clasificación de Raunkaier (1934) con la revisión de Ellemberg \& Mueller-Dombois (1967).

\section{RESULTADOS Y DISCUSIÓN}

\section{Fases fenomorfológicas de las especies estudiadas}

\section{Asparagus acutifolius L.}

Geófito, espinescente, de ramas perennes de hasta $1,5 \mathrm{~m}$ de altura. Crecimiento vegetativo del dolicoblasto de noviembre a enero, a partir de yemas situadas sobre el rizoma. Las nuevas ramas elongan y desarrollan ramas laterales de enero a junio y se lignifican durante el verano. Yemas florales de abril a junio, simultáneamente a la elongación de las ramas. Floración en junio. Formación del fruto de junio a finales de agosto y dispersión de septiembre a octubre. Las ramas pierden la capacidad fotosintética y se secan durante la primavera del siguiente año, permaneciendo sobre la planta de uno a dos años más, arrojandose generalmente en noviembre.

Brachypodium retusum (Pers.) Beauv.

Hemicriptófito cespitoso, semidecíduo de verano, de hasta $45 \mathrm{~cm}$ de altura, con ramas de comportamiento camefítico. Los braquiblastos se forman a partir de yemas vegetativas de la cepa basal y mantienen su crecimiento durante todo el año, excepto en verano. Las ramas dolicoblásticas, de comportamiento cametofítico, se forman a partir de los braquiblastos temporales de la cepa basal o a partir de yemas vegetativas laterales sobre los tallos del año anterior. El crecimiento vegetativo de los dolicoblastos es de octubre a mayo, con período de máxima elongación en abril. Formación de las yemas inflorenciales y floración en abril. El crecimiento del fruto es de finales de abril a finales de mayo y la dispersión en mayo. Las hojas de los dolicoblastos son arrojadas de junio a octubre.

Calicotome villosa (Poiret) Link

Nanofanerófito, decíduo de verano, espinescente, de hasta $2,5 \mathrm{~m}$ de altura. Crecimiento vegetativo de las hojas en octubre, sobre las ramas lignificadas de la primavera anterior, formando braquiblastos no espinescentes. Crecimiento vegetativo de los dolicoblastos de febrero a mediados de marzo, laterales sobre las axilas de las hojas formadas en octubre, formandose sobre ellos nuevos braquiblastos parciales, espinescentes. Las yemas florales se forman en febrero, sobre las nuevas ramas y de forma simultánea al desarrollo de las mismas. Floración en marzo. Crecimiento del fruto de marzo a finales de mayo. Dispersión de junio a septiembre. Lignificación de los dolicoblastos de finales de marzo a agosto. Caída completa de las hojas de mayo a junio.

\section{Ceratonia siliqua $\mathrm{L}$.}

Fanerófito, dióico, siempreverde, esclerófilo, de hasta $10 \mathrm{~m}$ de altura. Crecimiento vegetativo de los dolicoblasto de enero a mayo. Las nuevas hojas se forman en abril. La prefloración es en octubre y la floración en noviembre. Las inflorescencias se localizan en la base de las ramas formadas en la primavera anterior. El crecimiento del fruto es de noviembre a junio y su maduración de junio a agosto. La dispersión comienza en agosto pero pueden mantenerse hasta la siguiente primavera. Las hojas son arrojadas de junio a septiembre.

\section{Cistus albidus L.}

Caméfito sufrutescente, semidecíduo de verano, de hasta $1 \mathrm{~m}$ de altura. Crecimiento vegetativo de los dolicoblastos de octubre a junio, con máxima elongación y formación de hojas en enero y febrero. Los braquiblastos laterales se forman de febrero a junio y mantienen su crecimiento durante prácticamente todo el año, con una parada los meses de julio a septiembre. Los nuevos dolicoblastos se localizan bajo la inflorescencia del año anterior. Prefloración en febrero y floración en marzo. Crecimiento del fruto de marzo a junio. Dispersión de junio a diciembre. En julio y agosto se produce la caída de la mayor parte de las hojas formadas en invierno y de algunos de los braquiblastos, otros pueden permanecer sobre la planta más de un año.

\section{Cistus clusii Dunal}

Caméfito subfrutescente, semidecíduo de verano, de hasta $1 \mathrm{~m}$ de altura. Crecimiento vegetativo de los dolicoblastos de octubre a julio. En mayo se produce una rápida elongación de ramas 
y la formación de las hojas. A finales de junio, cuando el crecimiento ha terminando, se forman los braquiblastos, laterales en las axilas de las primeras hojas formadas del dolicoblasto, manteniendo su crecimiento hasta octubre. El desarrollo de las yemas florales laterales es de febrero a abril, sobre las ramas lignificadas que se formaron la primavera anterior, cuyas hojas basales comienzan a ser arrojadas. Floración en marzo. En abril, los frutos inician el crecimiento que finaliza a finales de agosto. La dispersión es de agosto a diciembre. Las hojas de los dolicoblastos caen de junio a finales de agosto.

\section{Cistus crispus L.}

Caméfito subfrutescente, semidecíduo de verano, de hasta $35 \mathrm{~cm}$ de altura. El crecimiento vegetativo de los dolicoblastos es de octubre a junio, con máxima elongación y desarrollo de hojas en marzo, mes en que también se forman las yemas preflorales laterales. Floración desde finales de marzo a mediados de abril. En abril y mayo crecen los braquiblastos laterales sobre las axilas de las nuevas hojas formadas, su crecimiento se mantiene prácticamente todo el año excepto en los meses de julio y agosto. La formación del fruto es de abril a junio y su dispersión de junio a septiembre. La mayor parte de las hojas son arrojadas desde finales de junio a finales de agosto.

\section{Cistus monspeliensis $\mathrm{L}$.}

Caméfito subfrutescente, semidecíduo de verano, de hasta $1 \mathrm{~m}$ de altura. En septiembre se inicia, lentamente, el crecimiento de los dolicoblastos en el extremo de las ramas del año anterior. En noviembre se produce una rápida elongación y la formación de las hojas. Desde finales de noviembre a marzo del siguiente año, se desarrollan los braquiblastos laterales sobre las ramas ya formadas, cuyo crecimiento se mantiene durante todo el año, ralentizandose de julio a noviembre. La prefloración y máxima floración son de febrero a marzo. El crecimiento del fruto es de marzo a junio. La caída de las hojas de las ramas formadas en otoño se realiza de forma progresiva de finales de abril a agosto. El fruto dispersa de junio a octubre.

\section{Daphne gnidium L.}

Nanofanerófito, siempreverde, con ramas de comportamiento hemicriptofítico que se renuevan a partir de la cepa basal, de hasta $1,5 \mathrm{~m}$ de altura. La duración media de cada rama es de cinco años, después es arrojada por la planta y renovada generalmente de diciembre a febrero. El crecimiento vegetativo del dolicoblasto, en el extremo de las ramas del año anterior, es de diciembre a junio con período de máxima elongación en abril. Un lento crecimiento vegetativo puede iniciarse a finales de octubre y mantenerse hasta diciembre. Las yemas preflorales comienzan a formarse en el extremo de las nuevas ramas desarrolladas en junio y florecen continuadamente hasta finales de octubre. El fruto se forma de agosto a noviembre y la dispersión es de septiembre a finales de noviembre. En julio y agosto son arrojadas algunas hojas de las ramas del invierno anterior.

\section{Helianthemum syriacum (Jacq.) Dum.}

Caméfito subfrutescente, semidecíduo de verano, de hasta $50 \mathrm{~cm}$ de altura. El crecimiento vegetativo de los dolicoblastos es de febrero a abril, con rápida elongación y formación de hojas en abril. Los dolicoblastos elongan a partir de braquiblastos laterales temporales de los extremos de las ramas lignificadas. Los braquiblastos crecen de septiembre a julio. Formación de las yemas preflorales en mayo. Floración en junio. Formación del fruto de junio a finales de julio; dispersión en julio y agosto. Las hojas de los braquiblastos y de los dolicoblastos son arrojadas en julio y agosto. Algunos braquiblastos permanecen sin elongar más de un año y después son arrojados.

\section{Helichrysum stoechas (L.) Moench}

Caméfito subfrutescente, semidecíduo de verano, de hasta $40 \mathrm{~cm}$ de altura. Crecimiento vegetativo de los dolicoblastos de octubre a enero, a partir de braquiblastos laterales situados sobre las ramas del año anterior, generalmente bajo los restos de las inflorescencias. El período de máxima elongación del dolicoblasto y formación de hojas es de enero a marzo. El crecimiento vegetativo de los braquiblastos se mantiene durante todo el año, con una parada de octubre a junio. La formación de yemas preflorales, terminales, es en marzo. Floración en abril sobre los nuevos tallos formados. Crecimiento del fruto de abril a mayo, dispersión de mayo a julio. De junio a agosto caen las hojas formadas en el invierno. 


\section{Juniperus oxycedrus L. subsp. oxycedrus}

Nanofanerófito, dioico, siempreverde, de hasta $3 \mathrm{~m}$ de altura, hojas esclerófilas. Crecimiento vegetativo de los dolicoblastos de octubre a julio, con rápida elongación de mayo a julio en los individuos femeninos y de mayo a septiembre en los masculinos. Los dolicoblastos crecen a partir de braquiblastos laterales, temporales y axilares sobre hojas de ramas del año anterior que inician su crecimiento en septiembre. Floración de noviembre a diciembre. Crecimiento de las piñas de diciembre a octubre del siguiente año. Dispersión de octubre a diciembre también, del siguiente año. Caída de las hoja de julio a septiembre.

\section{Lavandula stoechas $\mathrm{L}$.}

Caméfito subfrutescente, semidecíduo de verano, de hasta $60 \mathrm{~cm}$ de altura. Crecimiento vegetativo de los dolicoblastos a partir de braquiblastos laterales del año anterior, de octubre a junio, con máxima elongación de enero a mediados de febrero; no todos los dolicoblastos elongados formaran inflorescencias, algunos permanecen en crecimiento vegetativo y sobre ellos se formaran nuevos braquiblastos en la próxima estación de crecimiento. El crecimiento de los braquiblastos laterales se mantiene durante todo el año, excepto de mediados de julio a mediados de agosto que se ralentiza. No todos los braquiblastos elongan a dolicoblastos, algunos permanecen como tales alrededor de un año y posteriormente son arrojados por la planta. La formación de yemas inflorenciales terminales es en febrero sobre los nuevos tallos elongados en el pasado invierno. La floración es en marzo. Formación del fruto de abril a mayo y la dispersión de mayo a julio. En agosto se produce la caída parcial de las hojas.

\section{Myrtus communis L.}

Nanofanerófito, siempreverde, fragante, de hasta 2,5 m de altura, hojas semiesclerófilas. Crecimiento vegetativo del dolicoblasto de febrero a mayo y período de máxima elongación y formación de hojas en abril. Las nuevas ramas crecen sobre los extremos de las ramas lignificadas del año anterior. Las yemas florales, laterales, se forman en mayo en las axilas de las hojas de las nuevas ramas. Floración de junio a primeros de julio. Crecimiento del fruto de finales de junio a noviembre, dispersión de septiembre a diciembre. Las hojas, de las ramas lignificadas del año anterior, son arrojadas en julio y agosto.

\section{Olea europaea L. var. sylvestris Brot.}

Fanerófito, siempreverde, de hasta $5 \mathrm{~m}$ de altura, hojas esclerófilas. Crecimiento vegetativo de los dolicoblastos de noviembre a junio, con máxima elongación y formación de hojas en enero. Las nuevas ramas se forman en los extremos de las ramas lignificadas del año anterior. El crecimiento vegetativo de los braquiblastos, laterales de las ramas lignificadas de la estación anterior, y de las nuevas ramas se mantiene durante todo el año, excepto de julio a septiembre. Formación de yemas preflorales en abril, en las axilas de las hojas de las nuevas ramas. Floración en abril y mayo. La formación del fruto es de finales de mayo a noviembre y la maduración de septiembre a finales de noviembre. La dispersión es de septiembre a finales de diciembre. Las hojas son arrojadas de junio a agosto.

\section{Phillyrea angustifolia L.}

Fanerófito siempreverde, de hasta $2,5 \mathrm{~m}$ de altura, hojas semiesclerófilas. Crecimiento vegetativo de los dolicoblastos en mayo, con rápida elongación y formación de hojas en los extremos de las ramas lignificadas de la primavera anterior. Las yemas preflorales, laterales sobre las ramas del año anterior, se forman en marzo. Floración en abril. El fruto crece de abril a octubre. Dispersa de finales de julio a noviembre. Las hojas, formadas en mayo del año anterior, caen de agosto a noviembre.

\section{Phlomis lychnitis L.}

Caméfito subfrutescente, semidecíduo de verano, de hasta $30 \mathrm{~cm}$ de altura. Crecimiento vegetativo de los dolicoblasto de octubre a abril, con período de máxima elongación de febrero a abril. Los nuevos dolicoblastos se forman a partir de braquiblastos temporales terminales sobre la cepa basal o laterales sobre los restos basales de ramas lignificadas del año anterior. El crecimiento de los braquiblastos se mantiene durante todo el año, siendo más lento de finales de junio a finales de septiembre. No todos los braquiblastos elongan a dolicoblastos, algunos permanecen como tales y son arrojados por la planta tras una año de vida. Formación de yemas preinflorenciales simultánea a la finalización de la elongación. Floración en abril. 
La formación del fruto es de abril a junio. La dispersión es de mayo a septiembre. En junio y agosto parte de las hojas basales de los dolicoblastos son arrojadas.

\section{Phlomis purpurea $\mathrm{L}$.}

Caméfito frutescente, semidecíduo de verano, de hasta $1,5 \mathrm{~m}$ de altura. Crecimiento vegetativo de los dolicoblasto de octubre a febrero, con rápida elongación y formación de hojas de finales de enero a mediados de febrero. Las nuevas ramas se forman a partir de braquiblastos laterales situados en los extremos de las ramas lignificadas de la estación anterior, bajo los restos inflorenciales. Crecimiento de los braquiblastos axilares sobre las hojas del nuevo dolicoblasto, de febrero a junio y, tras una ralentización en los meses de verano, se reanuda nuevamente a finales de septiembre. Formación de las yemas inflorenciales en febrero, simultánea a la elongación final del dolicoblasto. Floración en marzo. Formación del fruto de marzo a finales de abril y dispersión de mayo a septiembre. En julio y agosto caen la mayor parte de las hojas de los dolicoblastos. Los braquiblastos laterales, de las partes basales de las ramas que no elongan a dolicoblastos, permanecen como tales todo un año.

\section{Pistacia lentiscus $\mathrm{L}$.}

Fanerófito monoico, siempreverde, de hasta $2,5 \mathrm{~m}$ de altura, hojas semiesclerófilas. El crecimiento de los dolicoblastos, con rápida elongación y formación de hojas, es en mayo en los extremos de las ramas lignificadas de la primavera anterior. En febrero, las yemas preflorales de las inflorescencias masculinas, se forman en las axilas de las hojas superiores de las ramas lignificadas. En marzo se forman las de las inflorescencias masculinas a lo largo de toda la rama. La floración de ambos tipos de inflorescencias es en abril. La formación del fruto se inicia en mayo y continua hasta agosto. Dispersión de septiembre a abril del siguiente año. Caída de hojas de junio a agosto.

\section{Pistacia terebinthus L.}

Fanerófito, decíduo de otoño, de hasta $3 \mathrm{~m}$ de altura. Crecimiento vegetativo del dolicoblasto simultáneo a la formación de yemas florales y floración de abril a finales de mayo. Las yemas florales, laterales en ramas lignificadas del año anterior, florecen aproximadamente un par de semanas antes del inicio del crecimiento de las yemas vegetativas apicales, las cuales originan nuevos dolicoblastos que elongan hasta que finaliza su crecimiento en junio. Crecimiento del fruto de finales de abril a junio, dispersando de finales de junio a primeros de septiembre. Caída de las hojas de mediados de septiembre a la primera quincena de octubre. El proceso de lignificación de las nuevas ramas termina en marzo. Las yemas florales y vegetativas permanecen inactivas, sobre las nuevas ramas formadas desde octubre a marzo.

\section{Quercus coccifera L.}

Nanofanerófito, siempreverde, de hasta $3 \mathrm{~m}$ de altura, hojas esclerófilas. Crecimiento vegetativo del dolicoblasto, con rápida elongación y formación de hojas, de octubre a diciembre. En abril, en las axilas de las hojas de las nuevas ramas formadas, se desarrollan las yemas preinflorenciales laterales; las femeninas en los extremos, las masculinas en la parte basal. Floración en abril-mayo. Las yemas vegetativas apicales crecen y pueden elongar cortamente en algunas ocasiones. Crecimiento del fruto de mayo a abril de la siguiente primavera. La dispersión es de agosto a noviembre del segundo año. Las hojas formadas en otoño se arrojan de junio a agosto, tras un año completo de su formación.

Quercus ilex L. subsp. ballota (Desf.) Samp.

Fanerófito siempreverde, de hasta $10 \mathrm{~m}$ de altura, hojas esclerófilas. Crecimiento vegetativo del dolicoblasto en octubre, con formación de nuevas hojas a partir de braquiblastos terminales situados en las ramas del año anterior. En febrero se forman las primeras yemas florales, que corresponden a las inflorescencias masculinas, localizadas en grupos en el extremo de las ramas o en las axilas de las hojas superiores de las mismas. La floración es en marzo, la de las inflorescencias masculinas es simultánea a la elongación de las nuevas ramas primaverales, en cuyos extremos se forman las yemas preflorales femeninas de floración inmediata. Crecimiento del fruto de mayo a agosto y dispersión de agosto a octubre. Caída de hojas de mayo a diciembre.

\section{Rhamnus velutinus Boiss.}

Nanofanerófito, semidecíduo de verano, espinescente, de hasta $2,5 \mathrm{~m}$ de altura. El crecimiento vegetativo de los braquiblastos se inicia en febrero, 
a partir de yemas vegetativas laterales sobre los dolicoblastos lignificados formados a finales de la primavera anterior. Elongación del braquiblasto y formación de hojas en marzo. Formación de yemas florales, en las axilas de las hojas, y floración en abril. En mayo, de forma simultánea al inicio del período de crecimiento del fruto, crecen los nuevos dolicoblastos con rápida elongación a partir de braquiblastos temporales. Lignificación y espinescencia en junio. El fruto continua madurando hasta finales de julio y dispersa de julio a septiembre. La dispersión coincide con la caída de las hojas de los braquiblastos que se mantiene de forma continuada hasta enero. Las yemas vegetativas de los nuevos braquiblastos permanecen inactivas, desde su formación, a finales de octubre hasta finales de enero del siguiente año.

\section{Rosmarinus officinalis L.}

Caméfito frutescente, siempreverde, fragante, de hasta 1,10 m de altura, hojas semiesclerófilas. Crecimiento vegetativo de los dolicoblastos de marzo a finales de agosto, con rápida elongación a finales de junio. Las nuevas ramas se forman en el extremo de las ramas del año anterior. Los braquiblastos laterales se desarrollan de febrero a junio en las axilas de las nuevas hojas formadas. Prefloración de octubre a diciembre y floración de noviembre a mayo. Las flores de otoño e invierno son axilares sobre las hojas de los dolicoblastos y las primaverales axilares, sobre las de los braquiblastos. Crecimiento vegetativo y formación de flores simultáneos de enero a mayo. El crecimiento de la mayor parte de los braquiblastos puede extenderse de uno a dos años. Formación del fruto de enero a junio. Dispersión de junio a julio. Caída de las hojas de los dolicoblastos en julio y agosto del siguiente año.

\section{Smilax aspera L.}

Caméfito, siempreverde, lianescente, hojas semiesclerófilas. Parte basal de la planta formando una cepa persistente. El crecimiento vegetativo del dolicoblasto se inicia en febrero, a partir de braquiblastos laterales temporales o a partir del extremo de las ramas del año anterior, la elongación y formación de nuevas hojas es de abril y mayo. Crecimiento secundario del dolicoblasto de septiembre a diciembre. Formación de las yemas preinflorenciales en octubre, en las axilas de las hojas de los tallos formados en el invierno anterior. Floración de octubre a noviembre. El fruto crece de noviembre a enero, una vez maduros, dispersan de finales de enero a febrero. El fin de la dispersión coincide con el inicio del desarrollo de nuevos dolicoblastos. Las hojas permanecen en la planta cerca de dos años y son arrojadas de junio a septiembre.

\section{Stipa tenacissima $\mathrm{L}$.}

Hemicriptófito cespitoso perenne, decíduo de verano, de hasta 1,50 m de altura. Los braquiblastos se forman a partir de yemas de la cepa basal persistente y crecen de octubre a junio. El crecimiento de los dolicoblastos es de noviembre a enero, a partir de braquiblastos. Los nuevos tallos elongan y desarrollan las hojas de finales de enero a febrero. La formación de las yemas inflorenciales y la floración es en febrero, en el extremo de los nuevos tallos formados. La formación del fruto es de marzo a abril y la dispersión en abril y mayo. Los dolicoblastos, una vez dispersado el fruto, se secan y caen de agosto a enero, otros pueden permanecer sobre ella más de una año. Las hojas basales de los tallos florales son arrojadas de mayo a septiembre.

\section{Teucrium fruticans $\mathrm{L}$.}

Nanofanerófito, semidecíduo de verano, de hasta $2,5 \mathrm{~m}$ de altura. Crecimiento vegetativo de los dolicoblastos de diciembre a febrero, con rápida elongación y formación de hojas en febrero. Los nuevos dolicoblastos crecen a partir de braquiblastos laterales situados en los extremos de las ramas lignificadas del invierno anterior. Los braquiblastos inician su crecimiento en octubre y lo mantienen hasta julio. Nuevos braquiblastos se desarrollan, en febrero, en las axilas de las hojas del nuevo dolicoblasto. No todos los braquiblastos laterales elongan a dolicoblastos, algunos permanecen como tales durante más de un año, tras el cual son arrojados por la planta. Las yemas preflorales se forman en febrero y son axilares sobre las hojas de las nuevas ramas, florecen de finales de febrero a marzo. Otras yemas florales pueden formarse también, sobre los braquiblastos laterales de las ramas del año anterior. La formación del fruto es de marzo a abril y la dispersión de abril a finales de mayo. Parte de las hojas de las nuevas ramas caen de junio a agosto. 
Teucrium lusitanicum Schreb. subsp. lusitanicum

Caméfito subfrutescente, semidecíduo de verano, de hasta $45 \mathrm{~cm}$ de altura. Crecimiento vegetativo de los dolicoblastos de octubre a junio, con elongación y formación de hojas de enero a marzo. Las nuevas ramas se forman a partir de braquiblastos laterales, temporales situados en las ramas lignificadas del invierno anterior. Los braquiblastos crecen durante todo el año con una parada de julio a finales de septiembre. La formación de las yemas preinflorenciales es en marzo y la floración en abril. La formación del fruto de abril a junio y la dispersión de junio a noviembre. Los braquiblastos, que no elongan a dolicoblastos, pueden permanecer como tales en la planta más de un año tras el cual caen. Las hojas son arrojadas de junio a agosto.

\section{Thymbra capitata (L.) Cav.}

Caméfito subfrutescente, semidecíduo de verano, de hasta $40 \mathrm{~cm}$ de altura. Crecimiento vegetativo de los dolicoblastos de diciembre a mayo, con período de máxima elongación y formación de hojas en abril-mayo. De forma simultánea a la elongación, se desarrollan los braquiblastos laterales axilares de las nuevas hojas formadas. Los braquiblastos continúan creciendo durante todo el año, excepto de agosto a noviembre. Los dolicoblastos se forman a partir de braquiblastos laterales situados en los extremos de las ramas lignificadas del año anterior, bajo los restos de la inflorescencia. Formación de yemas inflorenciales, terminales sobre las nuevas ramas formadas, en junio, floración de junio a primeros de julio. La formación del fruto es en junio y julio y la dispersión de julio a octubre. Caída de hoja en julio y agosto.

\section{Thymelaea hirsuta (L.) Endl.}

Nanofanerófito, semidecíduo de verano, de hasta $2 \mathrm{~m}$ de altura. Crecimiento vegetativo de los dolicoblastos de septiembre a junio, con rápida elongación de las ramas y formación de hojas en mayo-junio. Durante los meses de julio y agosto se mantiene el crecimiento de los braquiblastos formados de octubre a marzo. La prefloración es en diciembre y la floración en enero. El crecimiento del fruto es de febrero a abril y la dispersión en mayo. La caída de hoja, tiene lugar de julio a septiembre del segundo año.

\section{Thymus mastichina (L.) L. subsp. mastichina}

Caméfito subfrutescente, semidecíduo de verano, fragante, de hasta $45 \mathrm{~cm}$ de altura. Crecimiento vegetativo de los dolicoblastos, con elongación de nuevos tallos y formación de hojas, de enero a mayo, período máximo de elongación en abril. Crecimiento a partir de braquiblastos laterales primaverales situados bajo la inflorescencia del año anterior. Crecimiento de los braquiblastos durante todo el año, ralentizado desde finales de junio a finales de septiembre. Formación de yemas florales de mayo a junio. Floración, terminal sobre los nuevos tallos, de junio a julio. Crecimiento del fruto de finales de junio a finales de julio. Dispersión de julio a finales de agosto. Caída de hojas de los dolicoblastos, de junio a agosto.

\section{Ulex baeticus Boiss.}

Nanofanerófito, siempreverde, espinescente, de hasta $2 \mathrm{~m}$ de altura. Crecimiento vegetativo de los dolicoblastos de abril a mayo, las nuevas ramas crecen en la base de las ramas lignificadas del año anterior. Prefloración en diciembre, yemas laterales sobre las ramas formadas en la anterior primavera. Floración en enero. El inicio del crecimiento del fruto es de febrero a abril. Dispersión de finales de abril a mediados de junio. Lignificación de las ramas formadas en primavera de agosto a octubre, una vez que la planta ha terminado completamente la dispersión del fruto.

\section{Comportamiento fenológico y espectro biológico de la comunidad.}

El tipo biológico de una especie es el resultado de un ajuste morfológico a las condiciones ecológicas del medio donde se desarrolla (Cain, 1950; Van Rooyen et al., 1990). El análisis de los diferentes tipos biológicos del matorral estudiado (15 fanerófitos, 14 caméfitos, 2 hemicriptófitos y 1 geófito), revela el siguiente espectro: fanerófitos decíduos de invierno $(3,1 \%)$, fanerófitos siempreverdes $(18,75 \%)$, nanofanerófitos no espinescentes $(15,6 \%)$, nanofanerófitos espinescentes $(9,3 \%)$, caméfitos frutescentes $(21,8 \%)$, caméfitos 


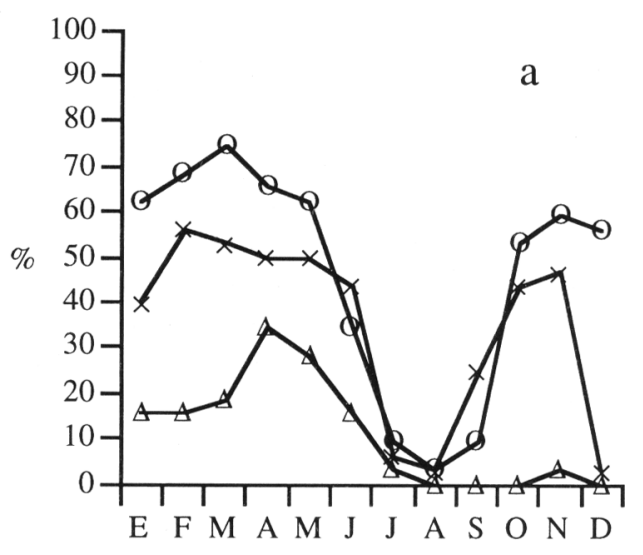

$\because \mathrm{AVD} \rightarrow \mathrm{AVB} \longrightarrow \mathrm{EL}$

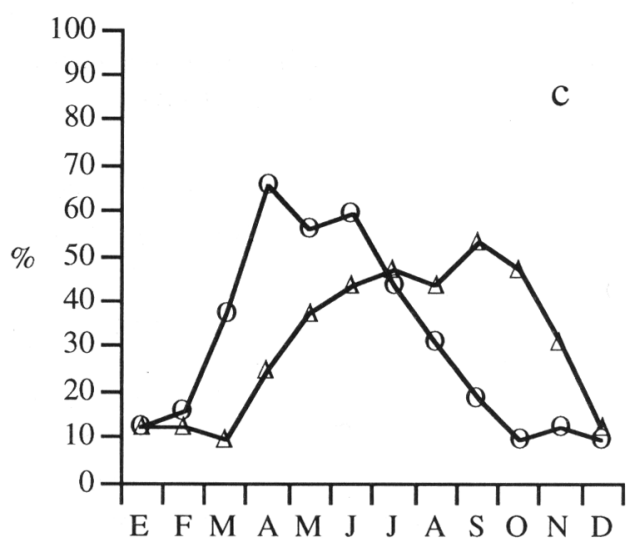

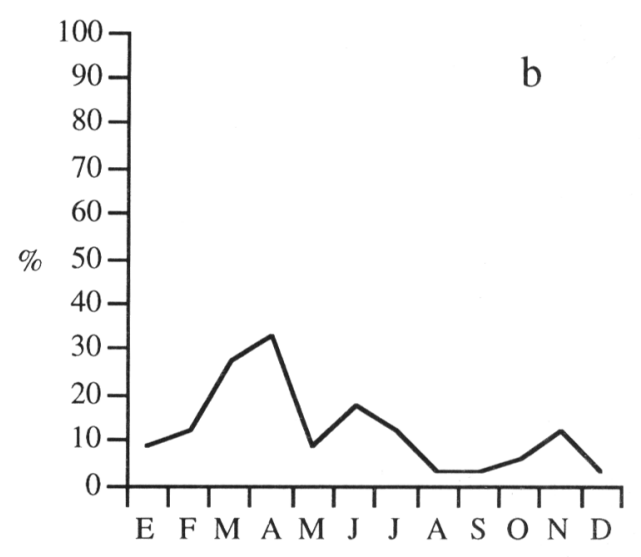

FL

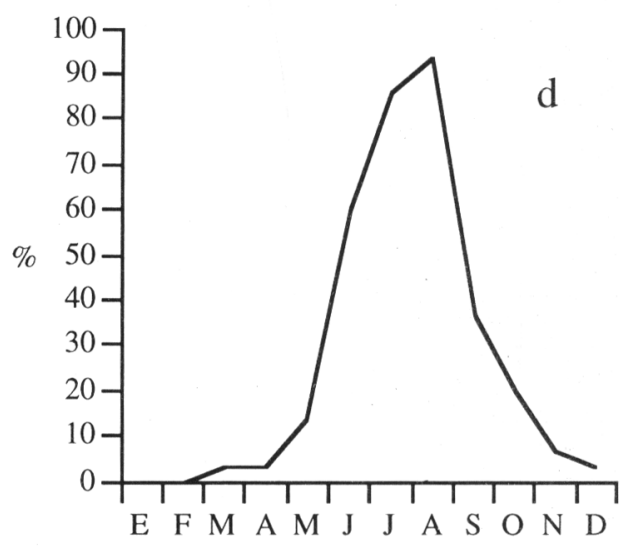

$\mathrm{CH}$

Figura 2. Perioricidad fenológica de la comunidad, media del periodo 1996-1997. a.- Actividad vegetativa. $(\mathrm{AVB}=$ Actividad Vegetativa del Braquiblasto); $(\mathrm{AVD}=$ Actividad Vegetativa del Dolicoblasto); (EL= Elongación del dolicoblasto). b.- Floración $(\mathrm{FL}=$ Floración $)$. c.- Crecimiento del fruto y dispersión. $(\mathrm{CF}=$ Crecimiento del Fruto); (DS= Dispersión). d.- Caída de la hoja. ( $\mathrm{CH}=$ Máxima caída de hoja del dolicoblasto). Phenological periodicity of the community, mean of the period 1996-1997. a.-Vegetative activity. $(A V B=$ Vegetative Activity of Brachyblasts $) ;(A V D=$ Vegetative Activity of Dolichoblasts $) ;(E L=$ Elongation of dolichoblasts). b.- Flowering. $(F L=$ Flowering). $c$. - Fruiting and Seed Dispersal. $(F R=$ Fruiting); (DS= Seed Dispersal). d.- Leaf-shedding. ( $\mathrm{CH}=$ Maximum leaf-shedding of dolichoblasts) 
subfrutescentes $(18,75 \%)$, caméfitos lianescentes $(3,1 \%)$, hemicriptófitos $(3,1 \%)$, hemicriptófitoscaméfitos $(3,1 \%)$ y geófitos $(3,1 \%)$.

El crecimiento vegetativo (actividad vegetativa de hojas y ramas) de la comunidad (fig. 2. a) se produce durante todo el año, variando el numero de especies que lo hacen en función de los cambios estacionales. El inicio de la primavera (marzo) y la finalización del otoño (noviembre) son los dos períodos estacionales que muestran una mayor actividad vegetativa. De las especies que crecen en marzo el $50 \%$ son caméfitos, el $40 \%$ fanerófitos y el 9\% hemicriptófitos y geófitos. En noviembre, la tasa más alta de crecimiento corresponde a los caméfitos con un $64 \%$, el $29 \%$ a los fanerófitos y el $5,8 \%$ a los hemicriptófitos. La emergencia de la hoja es en primavera para el $53 \%$ de los fanerófitos, en otoño para el $20 \%$ y en invierno-verano para un $6,6 \%$; en el caso de los caméfitos, el $71 \%$ presenta la emergencia de las hojas en primavera, el $35 \%$ en invierno y sólo el 7,1\% en otoño. El período de máxima elongación en la comunidad tiene lugar en el mes de abril, cuando la humedad del suelo es óptima como resultado de las lluvias de finales del invierno; el $45 \%$ de las especies que elongan en abril corresponden a los fanerófitos, el $36 \%$ a los caméfitos y el $9 \%$ a los geófitos.

La floración (fig. 2. b) muestra un pico en abril, mes en el que están en flor el $45 \%$ de los fanerófitos, el $36,3 \%$ de los caméfitos y el $9 \%$ de los hemicriptófitos. La primavera es la estación donde florecen la mayor parte de los fanerófitos $(66 \%)$; de ellos el $33 \%$ florecen sobre ramas lignificadas de la estación anterior y con inflorescencias laterales, el 33\% lo hace en inflorescencias terminales sobre nuevas ramas. El 20\% de los fanerófitos que florecen en invierno lo hacen sobre nuevas ramas. Del $64 \%$ de caméfitos que florecen en primavera, la práctica totalidad $(50 \%)$ lo hace sobre nuevas ramas y sólo un $14 \%$ sobre las ramas lignificadas de la estación anterior. El 28\% de los caméfitos florece en verano y el $14 \%$ en invierno.

El crecimiento (desarrollo) de los frutos (fig. 2. c) se produce fundamentalmente de febrero a junio con un máximo en marzo. La dispersión de los frutos y semillas se produce durante todo el año, con un pico de finales de verano a mediados de otoño.

El período de máxima caída de las hojas (fig. 2. d) se realiza durante los meses de julio y agosto, cuando la reserva de agua del suelo tiende a descender. De las especies siempreverdes que retienen la hoja más de 9 meses, el $80 \%$ son fanerófitos y el $20 \%$ caméfitos. De las que la retienen entre 6-9 meses, el $90 \%$ son caméfitos y el $10 \%$ fanerófitos. Las especies que retienen la hoja entre 4-6 meses corresponden a los decíduos de verano o de otoño.

\section{Estrategias fenomorfológicas de la actividad vegetativa en las especies estudiadas.}

En función de las estrategias fenomorfológicas de la actividad vegetativa, que las distintas especies de la comunidad estudiada presentan como adaptación a las peculiaridades del clima y microtopografía del hábitat, hemos distinguido los siguientes grupos: siempreverdes esclerófilas $(38,7 \%)$, decíduas de otoño $(6,2 \%)$, decíduas de verano $(6,4 \%)$ y semidecíduas de verano $(51,6 \%)$.

a. Siempreverdes (fig. 3. a). Especies esclerófilas tolerantes a los períodos de sequía (Mooney \& Kummerow, 1981) que mantienen estructuras verdes durante todo el año, sin una fase de pérdida generalizada de hojas, áridoactivas, por lo general con un solo período de crecimiento vegetativo primaveral (AVD1), que se caracteriza por una rápida de elongación (EL1) de las nuevas ramas y formación de hojas en el período previo a la sequía estival (Gratani \& Crescente, 1997; de Lillis \& Fontanella, 1992). En la comunidad estudiada 

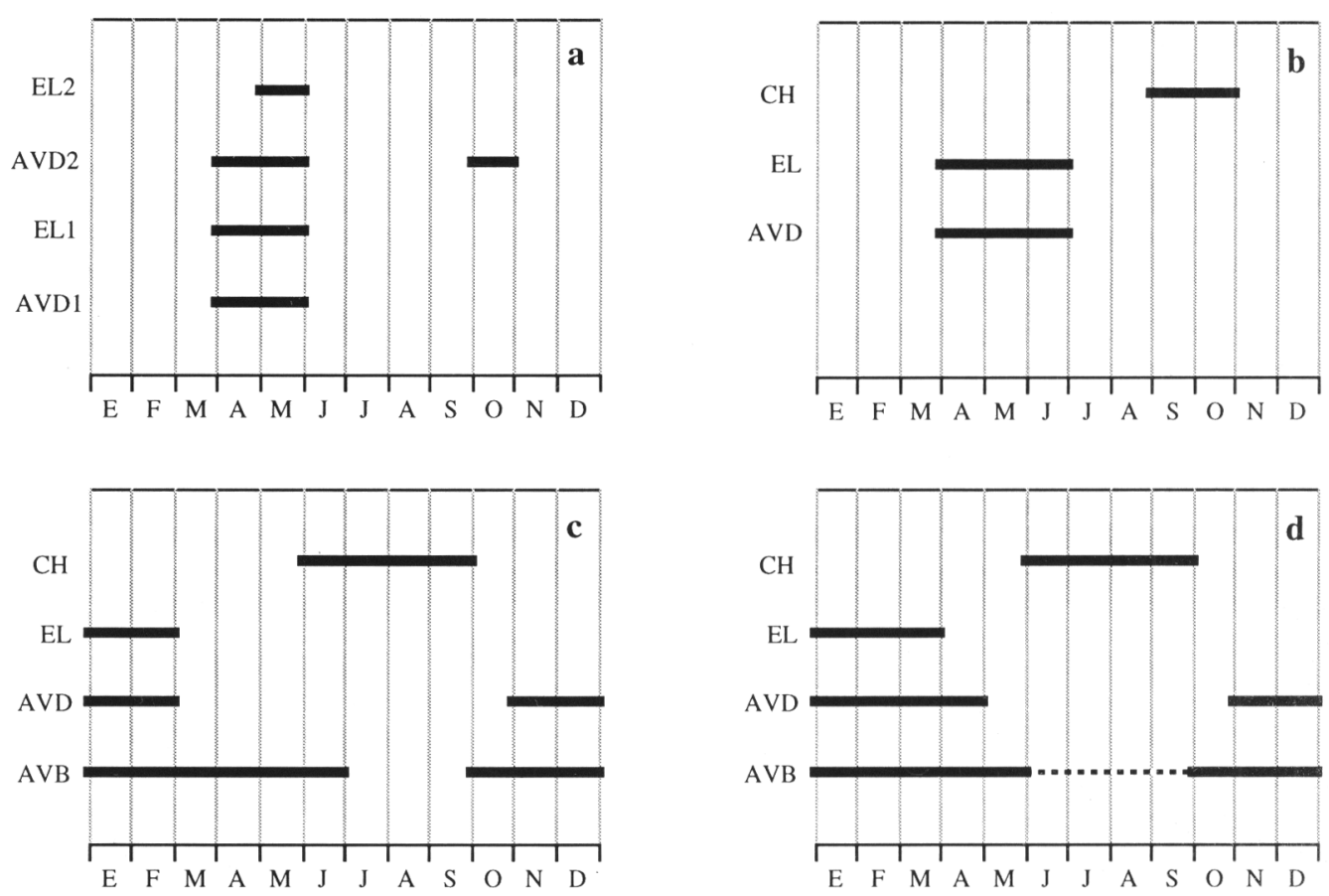

Figura 3. Estrategias fenomorfológicas anuales. a.- Modelo de comportamiento anual de las especies Siempreverdes Esclerófilas. b.- Modelo de comportamiento anual de las especies Deciduas de Otoño. c.Modelo de comportamiento anual de la especies Deciduas de Verano. d.- Modelo de comportamiento anual de las especies Semidecíduas de Verano. (AVB= Actividad Vegetativa del Braquiblasto. $(. .)=$. disminución del crecimiento; $\mathrm{AVD}=$ Actividad Vegetativa del Dolicoblasto; $\mathrm{EL}=$ Elongación del dolicoblasto; $\mathrm{CH}=$ Máxima Caída de Hoja del dolicoblasto). Annual phenomorphological strategies. a.-Annual phenological pattern in Evergreen Sclerophyllous species. b.- Annual phenological pattern in Autumn-deciduous species. c.- Annual phenological pattern in Summer-deciduous species. d.- Annual phenological pattern in Summer-semideciduous species. $(A V B=$ Vegetative Activity of Brachyblasts. $(\ldots)=$ decreasing of growth activity; $A V D=$ Vegetative Activity of Dolichoblasts; $E L=$ Elongation of dolichoblasts; $C H=$ Leafshedding of dolichoblasts).

pertenecen a este grupo fanerófitos o nanofanerófitos esclerófilos y semiesclerófilos que forman parte del estrato arbóreo, poco influenciados a los cambios de microtopografía, y dos caméfitos semiesclerófilos.

Con estas características encontramos un primer grupo de especies integrado por: Pistacia lentiscus, Phillyrea angustifolia, Juniperus oxycedrus subsp. oxycedrus, Myrtus communis, Ceratonia siliqua y Ulex baeticus, que presentan además una fase de floración previa al período de rápida elongación, con yemas florales laterales que se forman en las ramas lignificadas del año anterior. Otro grupo, formado por Olea europaea, Smilax aspera, Quercus ilex subsp. ballota y $Q$. coccifera, presentan un breve segundo período de crecimiento (AVD2) otoñal. En las especies de Quercus, la formación de yemas florales y la floración es simultánea con la elongación (EL2) de nuevas ramas. Rosmarinus officinalis se caracteriza por ser la única especie de la comunidad estudiada que mantiene el crecimiento y elongación de tallos durante el período de sequía. 
b. Decíduas de otoño. (fig. 3. b). Concentran su crecimiento vegetativo (AVD, EL) y reproductivo en primavera. En el resto de las estaciones no presentan ninguna actividad y pierden totalmente las hojas en otoño $(\mathrm{CH})$. Pistacia terebinthus es la única especie con esta estrategia en la zona de estudio.

c. Decíduas de verano. (fig. 3. c). No tolerantes a los períodos de sequía. Son especies herbáceas o leñosas, perennes árido-pasivas, que adoptan como estrategia un período de crecimiento (AVB, AVD) interrumpido por una fase estival sin actividad vegetativa durante la cual arrojan todas las hojas producidas en invierno $(\mathrm{CH})$. Pertenecen a este grupo: Stipa tenacissima y Calicotome villosa.

d. Semidecíduas de verano. (fig. 3. d). Especies semitolerantes al período de sequía, presentando en dicho período una significativa reducción de las partes verdes de la planta y una caída de la mayoría de las hojas producidas en invierno $(\mathrm{CH})$. Crecen durante todo el año (AVB), pero durante la sequía estival, la actividad vegetativa se reduce esencialmente a los braquiblastos, permitiendo una reducción de la transpiración (Zohary, 1962; Orshan, 1953). Es una estrategia, generalmente de caméfitos, intermedia entre las decíduas de verano y las siempreverdes esclerófilas, y ha sido citada por Correira et al., (1992), Arianoutsou-Faraggitaki \& Diamantopoulos (1985), Arianoutsou-Faragggitaki \& Maridis (1987) y de Lillis \& Fontanella (1992) en Portugal, Grecia e Italia respectivamente.

En la comunidad estudiada son mumerosas las especies con esta estrategia, pudiendo diferenciar distintos grupos en función de la adaptación al período seco, marcada fundamentalmente por la cantidad de masa foliar que se desprende. El grupo peor adaptado y con mayor pérdida de hojas está formado por caméfitos frutescentes como Teucrium fruticans, Cistus albidus, Cistus monspeliensis,
Helianthemun syriacum, Phlomis purpurea y Thymelea hirsuta y el nanofanerófito espinescente Rhamnus velutinus; en este grupo incluimos a Brachypodium retusum especie cuya forma de crecimiento es una transición entre hemicriptófito y caméfito típica de plantas cespitosas (Parolly, 1998). Con un mejor grado de adaptación y por tanto con una pérdida menos significativa de hojas encontramos un grupo de caméfitos subfrutescentes aromáticos y de altura inferior a $1 \mathrm{~m}$ como Thymbra capitata, Thymus mastichina subsp. mastichina, Lavandula stoechas, Teucrium lusitanicum subsp. Iusitanicum, Phlomis lychnitis, Helychrisum stoechas y Cistus crispus. Por último la especie mejor adaptada y con un comportamiento intermedio con las siempreverdes esclerófilas es Cistus clussii, con crecimiento durante todo el año, pero con una tasa de crecimiento muy rápida a finales de primavera, antes del período de sequía, como las típicas plantas esclerófilas.

\section{CONCLUSIONES}

Las especies tienden a explotar un amplio espectro de posibilidades para completar su ciclo de vida (Floret et al., 1984), utilizando los recursos del medio con la mínima competición entre las especies que coexisten en el mismo hábitat. En la comunidad estudiada se distinguen cuatro estrategias fenomorfológicas diferentes para las especies perennes, siendo las predominantes las siempreverdes esclerófilas y las semidecíduas de verano.

Las especies siempreverdes esclerófilas o semiesclerófilas, típicos elementos dominantes del maquis eu-mediterráneo (Quezel, 1985), son tolerantes a la sequía (Mooney \& Kummerov, 1981). En la comunidad estudiada, además de los típicos elementos eumeditarraneos, hemos incluido dentro de esta estrategia a otras especies esclerófilas como 
Myrtus communis, Ulex baeticus, Rosmarinus officinalis y Smilax aspera.

La degradación de la vegetación potencial, por la acción del hombre, facilita la invasión de caméfitos semidecíduos de verano que semitoleran la alta insolación. Son las típicas plantas mediterráneas (Dafni \& O’Toole, 1994; Herrera, 1986), con un máximo de actividad reproductiva y vegetativa que se concentra en abril-mayo, período de transición hacía la época de sequía. Su floración, a diferencia de las siempreverdes esclerófilas, se extiende al verano e invierno como ha sido observado en otras comunidades mediterráneas (Polunin \& Huxley, 1970; Specht et al., 1981; Lee, 1971).

Los decíduos de verano, Calicotome villosa y Stipa tenacissima, son los elementos con otro tipo de adaptación a las condiciones de máxima degradación de la zona, inactivos y sin ningún tipo de estructuras verdes en el período de sequía, concentran su actividad en otoño y primavera.

En cualquier caso el período de máximo estrés climático en la zona estudiada corresponde al período estival, con parada casi generalizada de la actividad vegetativa y pérdida de estructuras foliares como mecanismo morfo-fisiológico de adaptación.

\section{BIBLIOGRAFÍA}

ARIANOUTSOU-FARAGGITAKI, M. \& J. DIAMANTOPOLOUS -1985-Comparative phenology of five dominant plant species in maquis and phrygana ecosystems in Greece. Phyton 25(1): 77-85.

ARIANOUTSOU-FARAGGITAKI, M. \& TH. A. MARIDIS -1987- Observations on the phenology of two dominant plants of the Greek maquis. In: Plant response to strees. Funtional analysis in Mediterranean ecosystems. Tenhunen, J. D. Catarino, F. M., Lange, O. L. , and Oechel, W. C. (eds.): Springer-Verlag: New York-Heidelberg-Tokyo-Berlin: pp. 515-519.

ARROYO, J -1988- Atributos florales y fenología de la floración en matorrales del sur de España. Lagascalia 1(1): 43-78.

BERGMEIER, E. \& U. MATTHÄS -1996Phänologisches Spektrum einer kretischen phrygana. Tuxenia 16: 433-450.

BERGMEIER, E-1998. The phenological approach in Mediterranean landscape analysis. Phytocoenologia, 28(2): 145-156.

BERTILLER, M., A. M. BEESKOW \& F. CORONATO -1991- Seasonal environmental variation and plant phenology in arid Patagonia (Argentina). J. Arid. Env. 21: 1-11.

CABEZUdo, B., A.V. PÉREZ LATORRE, T. NAVARRO y J. M. NIETO-CALDERA -1993Estudios fenomorfológicos en la vegetación del sur de españa. II: Alcornocales mesomediterráneos. (Montes de Málaga, España). Acta Bot. Malacitana 18: 179-188.

CABEZUDO, B., T. NAVARRO, A.V. PÉREZ LATORRE, J. M. NIETO-CALDERA y G. ORSHAN - 1992- Estudios fenomorfológicos en la vegetación del sur de España. I. Cistus. Acta Bot. Malacitana 17: 229-237.

CAIN, S. A -1950- Life forms and phytoclimate. Bot. Rev. 16: 1-32.

CODY, M. L - 1986- Structural niches in plant communities. In: Diamond, J. \& Case, T.J. (Eds.), Community Ecology pp. 381-405. New York. Harper \& Row. 665 p.

CORREIRA, O. A., A. C. MARTINS \& F. M. CATARINO - 1992-Comparative phenology and seasonal foliar nitrogen variation in Mediterranean species of Portugal. Ecol. Medit. 18: 7-18.

DAFNI, A. \& C. O’TOOLE -1994.- Pollination syndromes in the Mediterranean: generalizations and peculiarities. In: M. Arianoutsou \& R. H. Groves, Plant-Animal Interactions in Mediterranean-Type ecosistems, Kluver Academic Publishers, Netherlands, pp. 125-135.

DIAMANTOPOULOS, J. \& N. S. MARGARIS 1981- Flowering times and flower colours in the flora of Greece. Phyton (Austria), 21:241244.

ELLEMBERG, H. \& D. MUELLER-DOMBOIS 1967- A key to Raunkaier plant life forms with revised subdivisions. Ver. Geobot. Inst. ETH, Stifg. Rübel, Zürich, 37: 56-73.

FLORET, C., E. Le FLOC'H, G. ORSHAN \& F. ROMANE -1984. Contribution à l'étude du cicle 
biologique de quelques espèces de la garrigue. Soc. Bot. Fr., 131, Actual. Bot.: 451-463.

GOMEZ-SAL, A., J. M. DE MIGUEL, M. A. CASADO \& F. D. PINEDA - 1986- Successional changes in the morphology and ecological reponses of a grazed pature ecosystem in Central Spain. Vegetatio 67: 33-44.

GRATANI, L. \& M. F. CRESCENTE -1997Phenology and leaf adaptative strategies of Mediterranean maquis plants. Ecol. Medit. 23(3/ 4): $11-19$.

HERRERA, J -1986- Flowering and fruiting phenology in the coastal shrublands of Doñana, South Spain. Vegetatio 68: 91-98.

HIDALGO, M. I. y B. CABEZUDO -1994Fenologia y volumen de floración del matorral de la Sierra de Mijas (Málaga, España). Acta Bot. Malacitana 19: 123-126.

ISH-SHALOM-GORDON, N - 1993.- Floristic composition and floral phenology of the Mediterranean batha of Ariel, Samaria. Vegetatio 109: 191-200.

KUMMEROV, J-1983-Comparative phenology of mediterranean-type plant communities. In: Kruger, F. J., Mitchell, D. T. \& Jarvis, J. U. M. (eds.). Mediterranean-type ecoystems: The Role of Nutrients. No. 43, Springer, Berlin, pp. 300317.

LAGUNA, E., M. GUARA y E. SÁNCHIZ -1986Estudios ecológicos de un transecto dunal. II. Fenología. Fol. Bot. Misc. 5: 105-116.

LEE, Y.N - 1971- Patterns of flowering periods in selected floras of the world. J. Korean Res. Inst. Better Living 6: 41-51.

LILLIS De, M. \& A. FONTANELLA -1992.Comparative phenology and growth in different species of the Mediterranean maquis of central Italy. Vegetatio 99-100: 83-96.

MONTENEGRO, G -1987-Quantification of Mediterranean plant Phenology and Growth. NATOASI series, vol. G 15. Plant response to stress. Tenhunen, J. D. Catarino, F. M. Lange, O. L., and Oechel, W. C (eds.). Springer-verlag: New York-Berlin-Heidelberg-Tokyo: 469-488.

MOONEY, H. A. \& J. KUMMEROW -1981Phenological development of plants in Mediterranean-climate regions. In: Ecosystems of the world 11, Mediterranean type shrublands. Elsevier: Amsterdam, pp. 249-255.

MORENO DURÁN, M. D., J. M. ORTEGA y M.
RODRÍGUEZ DE LOS SANTOS - 1997- Datos fenológicos de floración de algunas especies leñosas de la duna de Trafalgar (Barbate, Cádiz). Acta Bot. Malacitana, 21: 43-53.

NAVARRO, T., J. M. NIETO-CALDERA, A.V. PÉREZ LATORRE y B. CABEZUDO -1993Estudios fenomorfológicos en la vegetación del sur de España. III. Comportamiento estacional de una comunidad de badlands (Tabernas, Almería. España). Acta Bot. Malacitana 18: 189-198.

NIETO CALDERA, J. M., S. PÉREZ SANZ y B. CABEZUDO -1987- Datos sobre la vegetación dolomitícola del sector Rondeño. Lazaroa 10: $35-46$.

OECHEL, W. C., W. LAWRENCE, J. MUSTAFA \& J. MARTÍNEZ - 1981- Carbon allocation and utilization. In: Miller, P. C. (ed.) resource use by chaparral and matorral. A comparison of vegetation function in two Mediterranean type ecosystems. Springer-verlag: BerlinHeidelberg-New York: 151-186.

ORSHAN, G -1953- Notes on the application of Raunkiaers life forms in arid regions. Pales. $J$. Bot. (Jerusalem series), 6: 120-122.

ORSHAN, G -1989- Plant pheno-morphological studies in Mediterranean type ecosystems. Kluwer, Dordrecht.VI+404pp

ORSHAN, G., E. Le FLOC'H, A. Le ROUX \& G. MONTENEGRO -1988- Plant phenomorphology in Mediterranean type ecosystems. Time scales and water stres-proc. 5th Int. Conf. on Mediterranean Ecosystems. Di - Castri F., Floret, Ch., Ramball, S. Roy, J. (eds.) I.U.B.S. Paris: 111-123.

PAROLLY, G - 1998- Phytosociological studies on high mountain plant communities of the South Anatolian Taurus mountains 1. Scree plant communities (Heldreichietea): A synopsis. Phytocoenologia 28(2):233-284.

PÉREZ LATORRE, A. V., B. CABEZUDO, J. M. NIETO-CALDERA y T. NAVARRO -1995Caracterización fenológica y ecomorfológica de alcornocales andaluces (Málaga, España). Anal. Jard. Bot. Madrid 54: 554-560.

POLUNIN, O. \& A. HUXLEY -1970-Flowers of the Mediterranean. Chatto and Windus, London.

QUÉZEL, P - 1985- Definition of the Mediterranean region and the origin of the flora. In: GómezCampo, C (ed.). Plant Conservation In The 
Mediterranean Area. Dr. W. Junk Publishers, Dordrecht, pp. 9-43.

RAUNKAIER, C -1934- The life forms of plants and statical plant geography. Oxford.

SPECHT, R. L., R. W. ROGERS, \& A. J. M. HOPKINS -1981- Seasonal growth and flowering rhythms: Australian heathlands. In: Specht, R. L. (de.). Ecosystems of the world, vol. 9B, p. 5113. Elsevier, Amsterdam.

TRABAUD, L. \& B. de CHANTERAC - 1985- The influence of fire on the phenological behaviour of Mediterranean plant species in BasLanguedoc (France). Vegetatio 60: 119-130.

VAN ROOYEN, M. W., G. K. THERSON \& N.
GROBBERLAAR -1990- Life form and dispersal spectra of the flora of Namaqualand, S. Afr. J. Arid Env. 19: 133-145.

ZOHARY, M -1962- Plant Life of Palestine: Israel and Jordan. The Ronald Press, New York.

Aceptado para su publicación en Abril de 1998

Dirección de los autores. Dpto. Biología Vegetal. Facultad de Ciencias. Universidad de Málaga. 29071, Málaga. E-mail: tnavarro@uma.es 